\title{
A Data Analysis and Modelling Framework for the Evaluation of Interactive Information Retrieval
}

\author{
Ralf Bierig ${ }^{\star}$, Michael Cole, Jacek Gwizdka, and Nicholas J. Belkin \\ School of Communication and Information, Rutgers University, USA
}

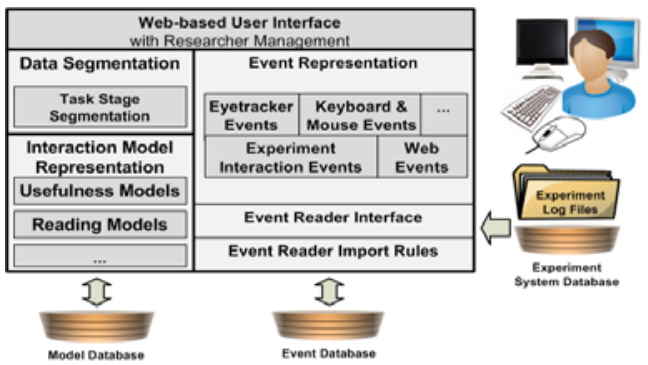

Fig. 1. System components of the data analysis and modelling framework

Over the last two decades, Interactive Information Retrieval (IIR) has established a new direction within the long tradition of IR that introduces the user at its center and poses new challenges for system evaluation. IR systems can improve performance by utilizing information about the entire interactive process of search. This approach has so far only been initially explored $[1,2]$ with much potential for the future. This demonstration describes an extensible data analysis and modelling framework that enables researchers to integrate, explore and analyze interactive experiment data obtained from task-based IIR experiments and build and test models of interactive user behavior. Figure 1 shows the framework components: The Event Representation integrates experiment data through the Event Reader Interface through a configurable set of Event Reader Import Rules into a unified and extensible event data structure. An extensible list of event types ensures that researchers can adapt and extend the framework to process data from a variety of IIR experiments on a single platform. Data Segmentation divides experiment data into semantic units guided by research hypotheses. A segmentation can for example differentiate interaction data based on users' current stage in the search task. The Interaction Model Representation processes (segmented) event sequences to test specific research hypotheses (e.g. identifying users' perceived usefulness of content or determining reading behavior). The Web-based User Interface extends the system to an online service where researchers can generate, inspect and share event representations and create data segmentations and interaction models. The framework is currently applied for the analysis of three IIR experiments in our research project. It will become an open source project that allows for wider public access within the research community.

\footnotetext{
* This work is supported, in part, by the Institute of Museum and Library Services (IMLS grant LG-06-07-0105-07).
}

C. Gurrin et al. (Eds.): ECIR 2010, LNCS 5993, pp. 673-674, 2010.

(C) Springer-Verlag Berlin Heidelberg 2010 


\section{References}

1. Belkin, N., Keynote: Some(what) Grand Challenges for Information Retrieval. In: 30th European Conference on Information Retrieval Research. Glasgow, Scotland, 2008.

2. Belkin, N., Bierig, R., Buscher, G., Van Elst, L., Gwizdka, J., Jose, J., Teevan, J.,: Workshop on Understanding the User. In: 32nd Annual ACM SIGIR Conference, Boston, USA, 2009.

\section{Requirements}

The Demonstration requires an internet connection and a large screen (19" or higher) to show the user interface in one-to-one discussions. A projector would be beneficial for larger audiences. A notebook is provided by the demonstrator. 


\section{Author Index}

Agarwal, Shivani 332

Alfonseca, Enrique 62

Alonso, Omar 13, 602, 667

Altingovde, Ismail Sengor 306

Aly, Robin 241

Anderka, Maik 640

Arampatzis, Avi 594

Araujo, Lourdes 26

Athanasakos, Konstantinos 217

Atilgan, Duygu 306

Azzopardi, Leif $153,204,357$

Baillie, Mark 432, 569

Balog, Krisztian 319

Bashir, Shariq 457

Becker, Steffen 668

Bedathur, Srikanta 13, 667

Belkin, Nicholas J. 673

Bellogín, Alejandro 382

Bennett, Paul N. 140

Berberich, Klaus 13, 623, 667

Bhattacharya, Pushpak 573

Bhole, Abhijit 573

Bierig, Ralf 673

Boudin, Florian 50

Bron, Marc 319

Broschart, Andreas

623

Cai, Deng 577

Cantador, Iván 420

Carman, Mark J. $\quad$ 432, 649

Castells, Pablo 382

Cerviño Beresi, Ulises 569

Chang, Hau-Wen 229

Chen, Long 370

Chen, Luke 394

Clements, Maarten 658

Cole, Michael 673

Collins, Michael 332

Collins-Thompson, Kevyn 140

Collins, Trevor 670

Cox, Ingemar 265

Crestani, Fabio 649

Dai, Na 127, 653

Daumé III, Hal 444
Davison, Brian D. 127, 653

de Jong, Franciska 153, 204

de Rijke, Maarten 191, 319

de Vries, Arjen P. 658

Doherty, Aiden 241

Efthimiadis, Efthimis N. 100

Eriksson, Gunnar 38

Fang, Hui 344

Ferrarotti, Flavio 281

Frieder, Ophir $\quad 483,636$

Frommholz, Ingo 615

Fuhr, Norbert 293, 544

Fu, Ruoxun 265

Glass, David H. 394

Goharian, Nazli 636

Gooda Sahib, Nuzhah 532

Gottron, Thomas 611

Gurevych, Iryna 598

Gurrin, Cathal 1

Gwizdka, Jacek 673

Hall, Keith 62

Hanjalic, Alan 645

Han, Jiawei 166

Han, Mei 581

Hansen, Lars Kai 265

Harvey, Morgan 432

Hauff, Claudia 153, 204

He, Tingting 370

He, Yulan 1

Hiemstra, Djoerd $153,204,241,556$

Huang, Xiangji 495

Hu, Biyun 619

Inches, Giacomo 649

Ingwersen, Peter 627

Jagarlamudi, Jagadeesh 444

Jambor, Tamas 407

Jin, Jianming 590

Jones, Gareth J.F. $\quad 520$

Jose, Joemon M. 217, 420 
Kabadjov, Mijail 662

Kamps, Jaap 556, 594

Kaptein, Rianne 556

Karlgren, Jussi 38

Kazai, Gabriella 1

Kelly, Liadh 520

Khapra, Mitesh M. 75

Kim, Hung-sik 229

Kim, Hyun Duk 166

Kim, Yunhyong 569

Knoth, Petr 670

Kriewel, Sascha 544

Kruschwitz, Udo 1

Lalmas, Mounia 615

Lapata, Mirella 12

Larsen, Birger 627

Larson, Martha 645

Lee, Dongwon 229

Lee, Jeongkyu 229

Lipka, Nedim 611

Little, Suzanne 1

Li, Zhoujun $\quad 495,619$

Lopez de Lacalle, Maddalen 586

Lu, Jinsong 577

Lund, Haakon 627

Luo, Jing 370

Lykke, Marianne

627

Macdonald, Craig $\quad 87,114$

Marín, Mauricio 281

Martin-Brualla, Ricardo 62

Martinez-Romo, Juan 26

McClean, Sally 394

Mendoza, Marcelo 281

Morin, Emmanuel 253

Moshfeghi, Yashar 615

Müller, Christof 598

Mundrawala, Jay 636

Nie, Jian-Yun 50

Ounis, Iadh $\quad 87,114$

Paşca, Marius 62

Papapetrou, Odysseas 293

Peña Saldarriaga, Sebastián

Peng, Jie 87, 114

Piwowarski, Benjamin 615
Poblete, Barbara 281

Poesio, Massimo 662

Potthast, Martin $\quad 631,640,668,672$

Pouliquen, Bruno 662

Raman, Karthik 573

Rauber, Andreas 457

Redpath, Jennifer 394

Reinders, Marcel J.T. 658

Robledo-Arnuncio, Enrique 62

Roelleke, Thomas 1

Rudinac, Stevan 645

Rüger, Stefan 1

Ruthven, Ian $\quad 432,532,569$

Sahlgren, Magnus 38

Sanderson, Mark 179

Santos, Rodrygo L.T. 87

Saralegi, Xabier 586

Schenkel, Ralf 508, 602, 623

Schütze, Hinrich 11

Serdyukov, Pavel 658

Shi, Lei 471

Shi, Lixin 50

Shokouhi, Milad 607

Siberski, Wolf 293

Sklavounou, Elsa 670

Smeaton, Alan 241

Smyth, Barry 10

Song, Dawei 569

Soo, Jason 483

Stamou, Sofia 100

Stathopoulos, Vassilios 217

Stein, Benno $\quad 631,640,672$

Steinberger, Josef 662

Steinberger, Ralf 662

Täckström, Oscar 38

Tang, Jiayu 179

Theobald, Martin 602

Thomas, Paul 607

Tombros, Anastasios 532

Trenkmann, Martin 631, 672

Tsagkias, Manos 191

Tu, Xinhui 370

Udupa, Raghavendra $\quad 75,573$

Ulusoy, Özgür 306 
Vallet, David 420

van Rijsbergen, Keith 1,615

Viard-Gaudin, Christian 253

Wang, Jun 407, 577, 619

Wang, Yaoshuang 653

Weerkamp, Wouter 191

Weikum, Gerhard 13,667

Xing, Xing 581

Xiong, Yuhong 590
Yang, Shengwen 590

Yee, Wai Gen 636

Yin, Xiaoshi 495

Zdrahal, Zdenek 670

Zhai, ChengXiang 166

Zhang, Dell 577

Zhang, Maoyuan 370

Zhang, Yi 581

Zheng, Wei 344

Zhu, Jianhan 265

Zuccon, Guido 357 